Extended Abstract

\title{
Methodology Shift in Information Studies
}

\author{
Yixin $\mathrm{ZHONG}^{*}$ \\ School of Computing \\ University of Posts and Telecommunications, Beijing 100876, China \\ e-mail: zyx@bupt.edu.cn
}

Tel: 8610-6228-1262

Accepted:

\section{Introduction}

Worldwide information studies has been so diversified nowadays that the unified theory of information science seems very hard to achieve within a foreseeable time frame. People with different backgrounds in academy may have different explanations for this diversity. To my understanding, however, the major factor responsible for the diversity is the improper employment of methodology in information studies.

\section{Methods}

As is well known, the studies of information science are very different from that of physical science. Yet, most of authors in information studies are still employing the methodology, which is only suitable for the studies of physical science and is featured by "divide and conquer", or reductionism. In the studies of information science, information plays the role of the lifeline of an information system. By employment of the method of "divide and conquer", the information lifeline will be cut into a number of smaller pieces and the properties of information system can never be recovered by summing up all the smaller pieces.

\section{Results and Discussion}

Therefore, information studies should experience a great shift in methodology, shifting from the traditional one in physical science to the new methodology, which is suitable in the studies of complex sciences and is featured by the information view, system view, ecology view, and the view of interaction between subject and object. This is because of the fact that information science is really a sort of complex science. 


\section{Conclusions}

This talk will provide an analysis for the methodology shift in information studies and will also provide an explanation to the general model for information studies derived from the methodology of complex science. The speaker believes that the new methodology and the model of information process derived from the methodology will be helpful and useful for information studies.

\section{Acknowledgments}

The author of this writing wants to express his thanks to the National Natural Science foundation of China for the valuable supporting to his research by projects granted.

\section{References and Notes}

1. Zhong, Y.X. Principles of Advanced Intelligence, Science Press: Beijing, China, 2014

(C) 2015 by the authors; licensee MDPI and ISIS. This abstract is distributed under the terms and conditions of the Creative Commons Attribution license. 